Evan Johnson, MD; John Stelzer, MD, MS; Andrew B. Romero, MD, MS; Joanne R. Werntz, MD University of Central Florida College of Medicine, Orlando (Drs. Johnson, Romero, and Stelzer); US Department of Veteran Affairs, Orlando VAMC, FL (Dr. Werntz); University of Connecticut, Department of Orthopaedic Surgery,

Farmington (Dr. Stelzer); Department of Orthopaedic Surgery and Biomedical Engineering, University of Tennessee-Campbell Clinic, Memphis (Dr. Johnson); Department of Orthopaedic Surgery, University of Central Florida/ HCA Healthcare GME

Consortium, Ocala (Dr. Romero)

\section{EEvanJohnsonOrtho@ gmail.com}

The authors reported no potential conflict of interest relevant to this article. doi: 10.12788/jfp.0239

\title{
Recognizing and treating trigger finger
}

\author{
This inflammatory condition can leave your patient in \\ pain and with impaired function. Here's what you need to \\ know about the diagnosis and Tx options to provide relief.
}

\section{PRACTICE \\ RECOMMENDATIONS}

> Recommend splinting as a first-line conservative treatment for trigger finger if there is not a fixed contracture. B

> Prescribe corticosteroids, which may completely resolve trigger finger in the majority of patients without diabetes. A

> Refer patients for surgical release if they do not respond to conservative management. The surgical success rate is as high as 99\%. (A)

Strength of recommendation (SOR)

A Good-quality patient-oriented evidence

B Inconsistent or limited-quality patient-oriented evidence

C Consensus, usual practice, opinion, disease-oriented evidence, case series

\section{CASE}

A 55-year-old right-hand-dominant woman presented to the clinic with a chief complaint of right ring finger pain and stiffness. There was no history of trauma or prior surgery. She had no tingling or numbness. She had a history of type 2 diabetes that was well controlled. She worked as a clerk for a government office for many years, and her painful, limited finger motion interfered with keyboarding and picking up items. Physical examination revealed tenderness to palpation over the palmar aspect of the metacarpophalangeal joint (MCPJ) of the ring finger with no other joint tenderness or swelling. When she made a fist, her ring finger MCPJ, proximal interphalangeal joint (PIPJ), and distal interphalangeal joint (DIPJ) locked in a flexed position that required manipulation to extend the finger. A firm mass was palpated in the palm with finger flexion that moved into the finger with extension.

0 tenosing tenosynovitis, also known as trigger finger (TF), is an inflammatory condition that causes pain in the distal palm and proximal digit with associated limited motion. The most commonly affected digits are the middle and ring fingers of the dominant hand. ${ }^{1}$ The disorder is particularly noticeable when it inhibits day-to-day functioning.

TF affects $2 \%$ to $3 \%$ of the general population and up to $20 \%$ of patients with diabetes. ${ }^{2,3}$ Patient age and duration of diabetes are commonly cited as contributing factors, although the effect of well-controlled blood glucose and A1C on the frequency and cure rate of TF has not been established. ${ }^{3,4} \mathrm{TF}$ is most commonly seen in individuals ages 40 to 60 years, with a 6 times' greater frequency in females than males. ${ }^{5}$

In the United States, there are an estimated 200,000 cases of TF each year, with initial presentation typically being to a primary care physician. ${ }^{6}$ For this reason, it is essential for primary care physicians to recognize this common pathology and treat symptoms early to prevent progression and the need for surgical intervention. 


\section{The pathology of trigger finger}

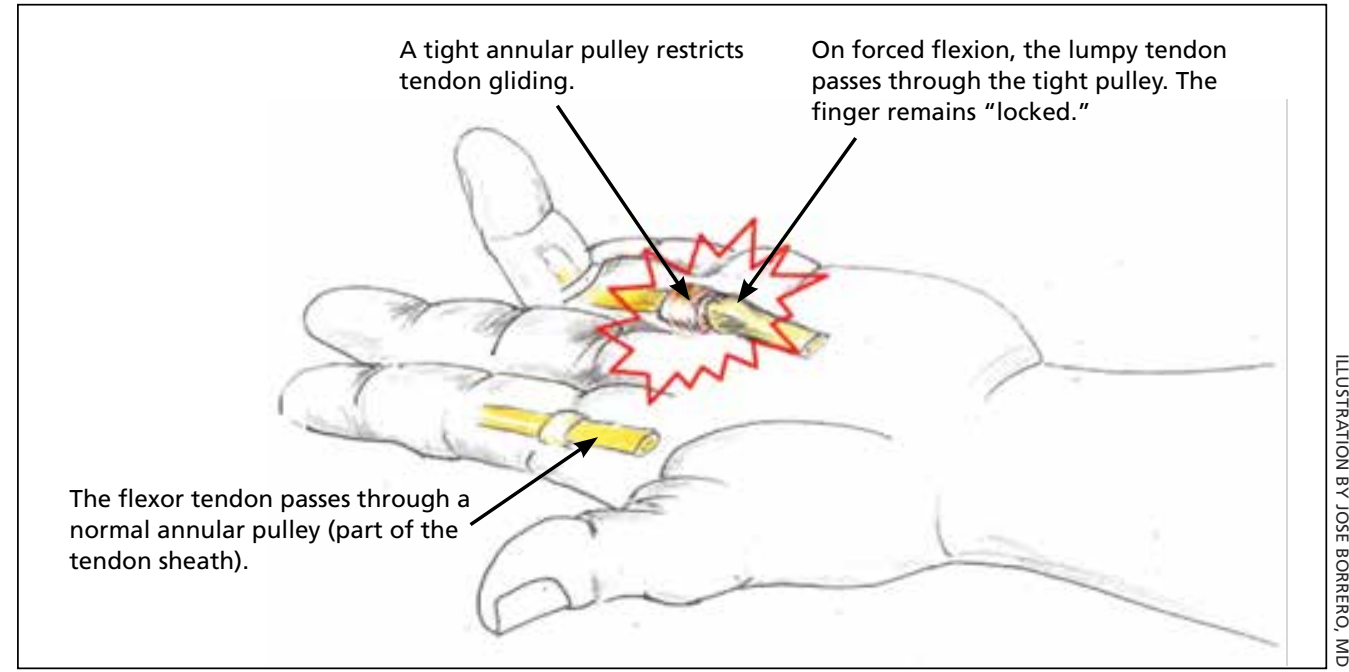

A stenotic tendon tunnel can lead to nodular formation of the flexor digitorum superficialis tendon. This results in difficulty with extension, significant pain, and a popping sensation when the nodule is forced under the pulley.

\section{An impaired gliding motion of the flexor tendons}

In each finger, a tendon sheath, consisting of 5 annular pulleys and 3 cruciate pulleys, forms a tunnel around the flexor digitorum profundus (FDP) and flexor digitorum superficialis (FDS). The tendon sheath allows for maximum force by eliminating bowstringing of the tendons when the digit is flexed. Deep to the tendons and surrounding the tendons is a synovial membrane that provides nutrition and reduces friction between the tendons and the tendon sheath. ${ }^{7}$

The FDP is longer and assists in flexion of the MCPJ and the PIPJ. It is the sole flexor of the DIPJ. The shorter FDS assists in flexion of the MCPJ and is the primary flexor of the PIPJ. The bifurcation of the shorter FDS tendon allows the longer FDP tendon to pass through to continue to its insertion on the distal phalanx.

In the thumb, the flexor pollicis longus (FPL) is the only flexor within its tendon sheath. The FPL assists in flexion of the MCPJ and flexes the thumb interphalangeal joint (IPJ). The intrinsic muscles (lumbricals and interossei) do not extend into the tendon sheath and do not contribute to TF.

I TF occurs when the tendon sheath, most commonly at the first annular pulley
(A1), or the flexor tendons thicken due to fibrocartilaginous metaplasia. This results in impaired gliding motion of the flexor tendons. ${ }^{8}$ The stenosed Al pulley can lead to pinching of the flexor tendons and cause the formation of a nodule on the FDS tendon at its bifurcation. ${ }^{9}$ The nodule of the FDS bifurcation moves proximal to the Al pulley when the finger is flexed. Upon extension, the tendon nodule may get caught on the Al pulley. This prevents smooth extension and is the source of pain and triggering (FIGURE 1). In a similar manner, thumb triggering is the result of a stenosed Al pulley creating a nodule on the FPL tendon, which prevents smooth gliding of the FPL.

\section{What you'll see}

TF is characterized by locking, popping, or clicking at the base of the finger or thumb. ${ }^{7,10} \mathrm{~A}$ small nodule may be palpated on the palmar aspect of the MCPJ when the finger is flexed. This nodule will then move distally when the finger is extended. Patients will present with the affected digit in a flexed position and will have difficulty extending the digit. In some cases, the patient may have to use the other hand to straighten the affected digit. In more severe cases, the digit may be fixed in a position of flexion or extension. The severity of
Trigger finger affects $2 \%$ to $3 \%$ of the general population and up to $20 \%$ of patients with diabetes. 
TABLE

Green's classification system ${ }^{11}$

\begin{tabular}{l|l}
\hline Grade & Symptoms \\
\hline I (pre-triggering) & Tenderness and pain at A1 pulley \\
\hline II (active) & Catching with active full extension \\
\hline IIIA (passive) & Catching requiring passive extension \\
IIIB (passive) & Inability to actively flex \\
\hline IV (contracture) & Catching with fixed flexion contracture of PIPJ \\
\hline
\end{tabular}

PIPJ, proximal interphalangeal joint.

triggering is commonly graded by the Green's classification system (see TABLE ${ }^{11}$ ).

\section{Is it Dupuytren contracture,} trigger finger, or something else?

The differential diagnosis for TF includes Dupuytren contracture, MCPJ sprain, calcific peritendinitis, flexor tenosynovitis, diabetic cheiroarthropathy (DCA), rheumatoid arthritis (RA), osteoarthritis (OA), and crystalline arthropathy (gout). ${ }^{5}$

I Dupuytren contracture is usually nonpainful and manifests with a palpable cord in the palm and a fixed flexion contracture that has progressed over time, with no history of catching.

I MCPJ sprain is diagnosed with tenderness of the MCPJ and a history of trauma.

I Calcific peritendinitis is characterized by pain, tenderness, and edema near a joint with calcified deposits seen on radiographs.

I Flexor tenosynovitis manifests with fusiform swelling of the digit, tenderness over the flexor tendon sheath, and pain with passive extension of the digit; it is more commonly associated with RA.

I DCA, RA, OA, and gout usually affect more than 1 digit. DCA is associated with both type 1 and type 2 diabetes and is characterized by thickened, waxy skin and painless, limited extension of the digits. RA and OA are diagnosed by medical history, lab work, and radiographs. Gout is diagnosed with lab work and aspiration of joint fluid.

A thorough history, physical exam, and review of radiographs must be performed to rule out these other disorders. Once the diagnosis of TF is made, available treatment options should be pursued.

\section{Treatment: A conservative} or surgical approach?

Current treatment options include both nonsurgical (conservative) and surgical interventions. Nonsurgical interventions include activity modification, splinting, and corticosteroid injections. While nonsteroidal anti-inflammatory drugs are commonly recommended to resolve the local inflammation secondary to triggering, there is no scientific evidence to support their use at this time. ${ }^{7}$ Surgical interventions, utilized in more severe cases or after conservative treatment has failed, include percutaneous and open release of the tendon sheath. ${ }^{2,7}$

\section{Conservative treatments}

I Splinting is only an option for digits that retain flexibility (Green's classification grades I, II, and III). The goal of splinting is to keep the affected digit in extension to avoid repeated friction between the tendon and the tendon sheath. ${ }^{12}$ This ideally allows any cartilaginous metaplasia or inflammation to resolve, subsequently alleviating symptoms. The recommended length of treatment with splinting ranges from 3 to 12 weeks, with an average of 6 weeks. ${ }^{1}$

Multiple studies have shown longterm alleviation of symptoms with the use of orthotic devices. A retrospective analysis found that $87 \%$ of patients who wore their PIPJ orthotic device both day and night for a minimum of 6 weeks required no further treatment at 1-year follow-up. ${ }^{13}$ In contrast, MCPJ splinting only at night has been shown to resolve symptoms in just $55 \%$ of patients after 6 weeks. ${ }^{14}$ From a practical standpoint, however, patients are more likely to be compliant with night-only splinting, making it a 
reasonable option. Splinting does remain efficacious for patients even after 6 months of symptomatology. ${ }^{15}$

Day and night splinting for approximately 8 weeks using a PIPJ orthotic could be considered as an effective first-line intervention. ${ }^{16}$ Notably, PIPJ splinting is more functional, as it allows motion of the MCPJ and DIPJ. There are several options available for PIPJ splints, including a stiff cushioned sleeve, a prefabricated plastic splint, and a large adhesive bandage.

An adjunct treatment to splinting is tendon-gliding exercises, including passive IPJ flexion, full finger flexion and extension, and hooking. ${ }^{13}$ Patients may remove the orthotic device to perform these exercises 3 times a day for 5 repetitions, as well as for activities that are not conducive to splinting. ${ }^{13}$

I Corticosteroid injections. Injections of a corticosteroid and $1 \%$ lidocaine in a 1:1 mixture for a total volume of $1 \mathrm{cc}$ can be inserted into the tendon sheath, Al pulley, or adjacent tissue. ${ }^{17}$ Steroid injections help to decrease inflammation and pain in the affected area, giving symptom relief lasting a few months in as many as $57 \%$ to $87 \%$ of patients. ${ }^{18}$

While the location of the injection has been debated, recent literature suggests that symptoms can be effectively alleviated regardless of the specific anatomic injection site, such as intra-sheath or extra-sheath (FIGURE 2) ${ }^{19}$ This allows flexibility for the clinician, as the injection does not have to be placed within the tendon sheath. Corticosteroids should not be injected into the tendon itself, and the needle tip should be slightly withdrawn if there is resistance while injecting. Patients who are averse to injections have been shown to benefit from needle-free jet lidocaine (J-tip) administration prior to the actual steroid injection. ${ }^{20}$

A randomized controlled trial comparing dexamethasone to triamcinolone injections found no difference in outcome at the 3 -month follow-up $(\mathrm{n}=84) .{ }^{17}$ This may suggest that the choice of corticosteroid is at the clinician's discretion. In terms of long-term efficacy of steroid injections, it has been shown that $70 \%$ of trigger digits had complete resolution of symptoms at a mean follow-up of 8 years after just 1 injection $(n=43){ }^{21}$
FIGURE 2

\section{A corticosteroid injection at} the Al pulley provides relief

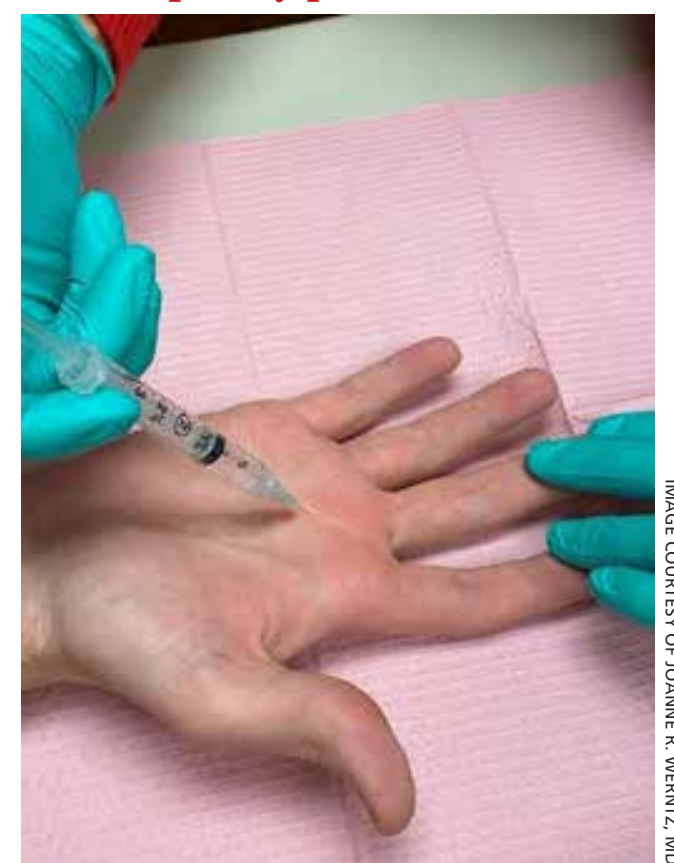

An injection of 1 cc of 1:1 dexamethasone and $1 \%$ lidocaine at the level of the A1 pulley is a common treatment modality for trigger finger. Efficacy has been shown, regardless of anatomic injection site (intra-sheath or extra-sheath).

Some patients, though, may require additional corticosteroid injections to maintain symptom control. If multiple injections are performed, they should not be given in intervals shorter than 4 months between treatments. ${ }^{5}$ Furthermore, steroids can be administered safely up to 3 times in the same digit before surgery is recommended..$^{22}$

A patient's options should be reconsidered if efficacy is not demonstrated with prior injections. Notably, a lower success rate has been shown in patients with type 2 diabetes (66\%) compared to those without diabetes (90\%), ${ }^{4,23}$ This difference in success rates is not well understood, as there is no causal relationship between well-controlled diabetes and $\mathrm{TF}^{4}$ Complications of corticosteroid injections include local pain, fat atrophy, and hypopigmentation at the site of the injection, as well as short-term elevations in blood glucose levels in patients with diabetes. ${ }^{5,24}$

Surgical correction (to be discussed) remains superior to steroid injections in 
FIGURE 3

\section{The A1 pulley release}

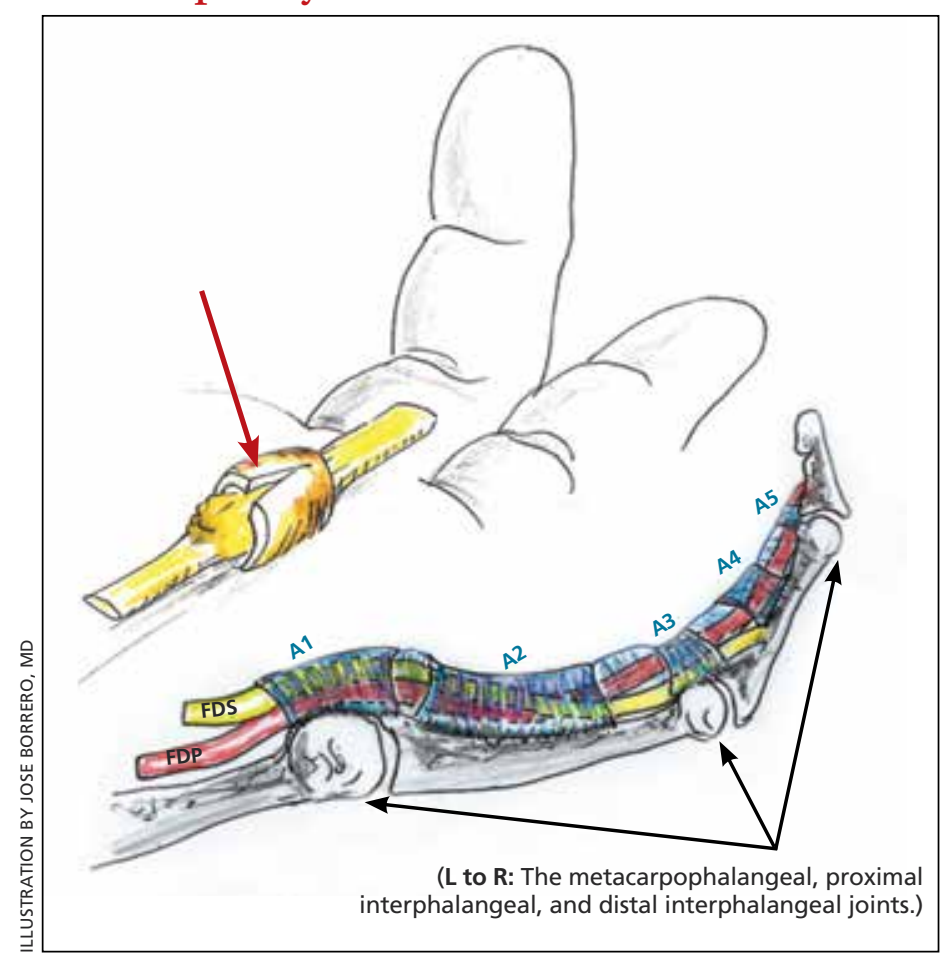

For definitive surgical management, the A1 pulley must be divided (red arrow) to relieve the obstruction and allow smooth flexion and extension.

FDP, flexor digitorum profundus; FDS, flexor digitorum superficialis.

terms of cure rate and resolution of symptoms. A randomized controlled trial $(\mathrm{n}=165)$ found that an injection-only group reported $86 \%$ and $49 \%$ success at 3 -month and 12-month follow-up, respectively, compared to $99 \%$ success at both 3 - and 12-month follow-up for the surgical group. Further, at 12-month follow-up, the median pain scores were significantly higher in the injection group (3; range, 1-9) than in the surgery group ( 1 ; range, 1-7). ${ }^{25}$ If conservative treatment modalities lead to unresolved symptoms or recurrence, referral to a hand specialist for surgery is recommended.

\section{Surgical treatments} in an office setting

Procedures for TF can be safely performed under conscious sedation or local anesthesia, with or without a tourniquet. ${ }^{26}$ Wide-awake procedures with local anesthesia and no tourniquet (WALANT) can be performed in an office-based procedure room rather than the operating room. This increases efficiency for the surgeon, reduces the amount of preparation and recovery time for the patient, and helps to keep costs down.

I Percutaneous release involves the insertion of a 16-gauge hypodermic needle into the affected Al pulley. The needle is used to fray and disrupt the pulley by moving the needle tip over the fibrotic Al pulley.

However, it is not without possible complications ${ }^{27}$ Inadvertent A2 pulley damage is particularly troublesome, as it leads to "bowstringing" or protrusion of the flexor tendon into the palm upon flexion. This can cause pain and failure to fully extend or flex the finger. ${ }^{10}$ Because the anatomy is not well visualized during the percutaneous approach, incomplete release, neurovascular injury, and iatrogenic injury to the A2 pulley or deep tendon may occur. ${ }^{28}$ Ultrasound-guided percutaneous release techniques have shown effective clinical outcomes with minimal complications compared to nonguided percutaneous release techniques. ${ }^{29,30}$

I Open release is the gold standard surgical treatment for trigger finger (FIGURE 3). A small incision $(1-2 \mathrm{~cm})$ is made directly over or proximal to the Al pulley in the distal palmar crease at the base of the affected digit. After blunt dissection through the subcutaneous tissue, the Al pulley is sharply incised. An open approach has the clear benefit of avoiding the digital neurovascular bundles, as well as visualizing the resolution of triggering upon flexion and extension prior to closure. The WALANT procedure has the advantage of allowing the awake patient to actively flex and extend the digit to determine if the $\mathrm{Al}$ release has been successful prior to closure of the incision.

I Outcomes and complications of surgery. A recent systematic review and metaanalysis has shown percutaneous techniques to be successful in $94 \%$ of cases. ${ }^{27}$ The success rate of open surgery has been reported at $99 \%$ to $100 \%$ at varying follow-up intervals up to 1 year. ${ }^{25,30,31}$ The complication rate for percutaneous release (guided and nonguided) was calculated at $2.2 \%(\mathrm{n}=2114) .{ }^{27} \mathrm{In}$ another study, the overall complication rate of open releases was calculated at $1 \%(\mathrm{n}=999) .{ }^{32}$ When 
comparing percutaneous release (guided and nonguided) and open release, a meta-analysis found no significant difference in complication rate $(\mathrm{RR}=0.84)$ or failure rate $(\mathrm{RR}=0.94) .^{32}$

Several risk factors have been associated with postoperative surgical infection, including recent steroid injection $(<80 \mathrm{~d})$, smoking status, increasing age, and pre-operative use of lidocaine with epinephrine. ${ }^{33}$ Open release has been shown to be an effective and safe treatment modality for patients with and without diabetes alike. ${ }^{34}$ Overall, definitive surgical correction has been demonstrated to be superior to conservative measures due to a significantly lower rate of recurrence..$^{35}$

\section{CASE}

Given the patient's presentation with triggering of the digit, tenderness over the A1 pulley, and lack of trauma history, we diagnosed trigger finger in this patient. Potential treatments included splinting, corticosteroid injections, and surgery. After discussion of the risks and benefits of each treatment option, the patient elected to undergo a corticosteroid injection. She was also given a neoprene finger sleeve to wear every night, and in the daytime when possible.

At 12-week follow-up, she noted early improvement in her triggering, which had since recurred. Due to her history of diabetes, the patient was then referred for surgery. She had an open release under local anesthesia. The surgery was uncomplicated, and the abnormality was corrected. At the patient's 1-year postoperative follow-up visit, there was no evidence of recurrence, and she had regained full active and passive range of motion of her finger.

JFP

\section{ACKNOWLEDGEMENTS}

The authors wish to thank Jose Borrero, MD, for contributing his time and creative talents to produce the illustrations in this article.

\section{CORRESPONDENCE}

Evan P. Johnson, MD; 506 South Greer Street, Memphis, TN 38111; EvanJohnsonOrtho@gmail.com

\section{REFERENCES}

1. Lunsford D, Valdes K, Hengy S. Conservative management of trigger finger: a systematic review. J Hand Ther. 2019;32:212-221. doi: 10.1016/j.jht.2017.10.016

2. Makkouk AH, Oetgen ME, Swigart CR, et al. Trigger finger: etiology, evaluation, and treatment. Curr Rev Musculoskelet Med. 2008;1:92-96. doi: 10.1007/s12178-007-9012-1

3. Fitzgibbons PG, Weiss AP. Hand manifestations of diabetes mellitus. J Hand Surg Am. 2008;33:771-775. doi: 10.1016/ j.jhsa.2008.01.038
4. Junot HSN, Anderson Hertz AFL, Gustavo Vasconcelos GR, et al. Epidemiology of trigger finger: metabolic syndrome as a new perspective of associated disease. Hand $\left(\begin{array}{ll}N & Y\end{array}\right)$. 2019:1558944719867135. doi: 10.1177/1558944719867135.

5. Matthews A, Smith K, Read L, et al. Trigger finger: an overview of the treatment options. JAAPA. 2019;32:17-21. doi: 10.1097/01. Jaa.0000550281.42592.97

6. Pencle FJ, Waheed A, Molnar JA. Trigger thumb. StatPearls [Internet]. StatPearls Publishing; 2020. www.ncbi.nlm.nih.gov/books/ NBK441854/

7. Giugale JM, Fowler JR. Trigger finger: adult and pediatric treatment strategies. Orthop Clin North Am. 2015;46:561-569. doi: 10.1016/j.ocl.2015.06.014

8. Bianchi S, Gitto S, Draghi F. Ultrasound features of trigger finger: review of the literature. J Ultrasound Med. 2019;38:3141-3154. doi: $10.1002 /$ jum.15025

9. Chuang XL, Ooi CC, Chin ST, et al. What triggers in trigger finger? The flexor tendons at the flexor digitorum superficialis bifurcation. J Plast Reconstr Aesthet Surg. 2017;70:1411-1419. doi: 10.1016/j.bjps.2017.05.037

10. Ryzewicz M, Wolf JM. Trigger digits: principles, management, and complications. J Hand Surg Am. 2006;31:135-146. doi: 10.1016/j.jhsa.2005.10.013

11. Chapter 56: Tendinoapthy. In: Wolfe SW, Peterson WC, Kozin SH, Cohen MS. Green's Operative Hand Surgery. Vol 2. 7th ed. Elsevier; 2017: 1904-1925.

12. Tarbhai K, Hannah S, von Schroeder HP. Trigger finger treatment: a comparison of 2 splint designs. J Hand Surg Am. 2012;37: 243-249, 249.e241. doi: 10.1016/j.jhsa.2011.10.038

13. Valdes K. A retrospective review to determine the long-term efficacy of orthotic devices for trigger finger. J Hand Ther. 2012;25: 89-95. doi: 10.1016/j.jht.2011.09.005

14. Drijkoningen T, van Berckel M, Becker SJE, et al. Night splinting for idiopathic trigger digits. Hand (N Y). 2018;13:558-562. doi: $10.1177 / 1558944717725374$

15. Colbourn J, Heath N, Manary S, et al. Effectiveness of splinting for the treatment of trigger finger. J Hand Ther. 2008;21:336-343. doi: 10.1197/j.jht.2008.05.001

16. Teo SH, Ng DCL, Wong YKY. Effectiveness of proximal interphalangeal joint-blocking orthosis vs metacarpophalangeal joint-blocking orthosis in trigger digit management: A randomized clinical trial. J Hand Ther. 2018;32:444-451. doi: 10.1016/ j.jht.2018.02.007

17. Ring D, Lozano-Calderon S, Shin R, et al. A prospective randomized controlled trial of injection of dexamethasone versus triamcinolone for idiopathic trigger finger. $J$ Hand Surg Am. 2008;33:516-522; discussion 523-514. doi: 10.1016/ j.jhsa.2008.01.001

18. Fleisch SB, Spindler KP, Lee DH. Corticosteroid injections in the treatment of trigger finger: A level I and II systematic review. J Am Acad Orthop Surg. 2007;15:166-171. doi: 10.5435/00124635200703000-00006

19. Shinomiya R, Sunagawa T, Nakashima Y, et al. Impact of corticosteroid injection site on the treatment success rate of trigger finger: a prospective study comparing ultrasound-guided true intra-sheath and true extra-sheath injections. Ultrasound Med Biol. 2016;42:2203-2208. doi: 10.1016/j.ultrasmedbio.2016.05.015

20. Earp BE, Stanbury SJ, Mora AN, et al. Needle-free jet lidocaine administration for preinjection anesthesia in trigger finger injection: a randomized controlled trial. J Hand Surg Am. 2017;42: 618-622. doi: 10.1016/j.jhsa.2017.05.001

21. Castellanos J, Munoz-Mahamud E, Dominguez E, et al. Longterm effectiveness of corticosteroid injections for trigger finger and thumb. J Hand Surg Am. 2015;40:121-126. doi: 10.1016/ j.jhsa.2014.09.006

22. Dala-Ali BM, Nakhdjevani A, Lloyd MA, et al. The efficacy of steroid injection in the treatment of trigger finger. Clin Orthop Surg. 2012;4:263-268. doi: 10.4055/cios.2012.4.4.263

23. Griggs SM, Weiss AP, Lane LB, et al. Treatment of trigger finger in patients with diabetes mellitus. J Hand Surg Am. 1995;20:787-789. doi: 10.1016/s0363-5023(05)80432-0

24. Stepan JG, London DA, Boyer MI, et al. Blood glucose levels in diabetic patients following corticosteroid injections into the hand and wrist. J Hand Surg Am. 2014;39:706-712. doi: 10.1016/ j.jhsa.2014.01.014

25. Hansen RL, Sondergaard M, Lange J. Open surgery versus ultrasound-guided corticosteroid injection for trigger finger: a randomized controlled trial with 1-year follow-up. J Hand Surg Am. 2017;42:359-366. doi: 10.1016/j.jhsa.2017.02.011
While NSAIDs are commonly recommended to resolve the local inflammation secondary to triggering, there is no scientific evidence to support their use. 
26. Mohd Rashid MZ Sapuan I, Abdullah S. A randomized controlled trial of trigger finger release under digital anesthesia with (WALANT) and without adrenaline. J Orthop Surg (Hong Kong). 2019;27:2309499019833002. doi: 10.1177/ 2309499019833002

27. Zhao J-G, Kan S-L, Zhao L, et al. Percutaneous first annular pulley release for trigger digits: a systematic review and meta-analysis of current evidence. J Hand Surg Am. 2014;39:2192-2202. doi 10.1016/j.jhsa.2014.07.044

28. Guler F, Kose O, Ercan EC, et al. Open versus percutaneous release for the treatment of trigger thumb. Orthopedics. 2013;36: e1290-1294. doi: 10.3928/01477447-20130920-22

29. Wu KC, Chern TC, Jou IM. Ultrasound-assisted percutaneous trigger finger release: it is safe [letter]. Hand ( $N$ Y). 2009;4:339. doi: 10.1007/s11552-009-9179-6

30. Nikolaou VS, Malahias M-A, Kaseta M-K, et al. Comparative clinical study of ultrasound-guided Al pulley release vs open surgical intervention in the treatment of trigger finger. World J Orthop.
2017;8:163-169. doi: 10.5312/wjo.v8.i2.163

31. Lim M-H, Lim K-K, Rasheed MZ, et al. Outcome of open trigger digit release. J Hand Surg Eur Vol. 2007;32:457-459. doi: 10.1016/ j.Jhsb.2007.02.016

32. Wang J, Zhao J-G, Liang C-C. Percutaneous release, open surgery, or corticosteroid injection, which is the best treatment method for trigger digits? Clin Orthop Relat Res. 2013;471:1879-1886. doi: 10.1007/s11999-012-2716-6

33. Ng WKY, Olmscheid N, Worhacz K, et al. Steroid injection and open trigger finger release outcomes: a retrospective review of 999 digits. Hand $(N Y)$. 2018;15:399-406. doi: $10.1177 / 1558944718796559$

34. Ho SWL, Chia CY, Rajaratnam V. Characteristics and clinical outcomes of open surgery for trigger digits in diabetes. J Hand Microsurg. 2019;11:80-83. doi: 10.1055/s-0038-1670927

35. Sato ES, dos Santos JB, Belloti JC, et al. Percutaneous release of trigger fingers. Hand Clin. 2014;30:39-45. doi: 10.1016/ j.hcl.2013.08.017

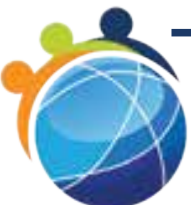

\section{MEDJOBNETWORK, $\mathrm{COM}$}

Physician $\bullet$ NP/PA Career Center

\section{The first mobile job board for Physicians, NPs, and PAs}

Mobille Job Searches - access MedJobNetwork.com on the go from your smartphone or tablet

Advanced Search Capabilities - search for jobs by specialty, job title, geographic location, employer, and more

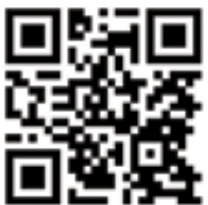

Scan this QR code

to access the mobile version

of MedJobNetwork.com 